\title{
Successful percutaneous coronary intervention during cardiac arrest with use of an automated chest compression device: a case report
}

This article was published in the following Dove Press journal:

Therapeutics and Clinical Risk Management

II April 2014

Number of times this article has been viewed

\section{Berglind Libungan \\ Christian Dworeck \\ Elmir Omerovic}

Department of Cardiology, Sahlgrenska University Hospital, Göteborg, Sweden
Correspondence: Berglind Libungan Department of Cardiology, Sahlgrenska University Hospital, Blå Stråket 3, våning I, 4I345 Göteborg, Sweden Email berglind.libungan@vgregion.se
Abstract: Ventricular tachycardia or fibrillation (VT/VF) in patients with ST-elevation myocardial infarction (STEMI) is associated with poor prognosis. Performing manual chest compressions is a serious obstacle for treatment with percutaneous coronary intervention (PCI). Here we introduce a case with refractory VT/VF where the patient was successfully treated with an automated chest compression device, which made revascularization with PCI possible.

Keywords: PCI, LUCAS, STEMI, automatic chest compressions, ventricular fibrillation, mechanical CPR

\section{Introduction}

Cardiac arrest is a major public health problem, which results in approximately 275,000 patients being treated for out-of-hospital cardiac arrest by the emergency medical services in Europe each year, of which approximately only $10 \%$ survive to hospital discharge. ${ }^{1}$

Closed chest compressions are one of the most important elements of cardiopulmonary resuscitation and when performed correctly, they increase coronary perfusion pressure. Coronary perfusion pressure $>15 \mathrm{mmHg}$ elevates the possibility of obtaining the return of spontaneous circulation following defibrillation. ${ }^{2}$ Goals of excellent chest compressions have been published in the guidelines. ${ }^{3}$

However, there are several drawbacks to performing manual chest compressions: inter- and intra-rescuer variability of compression quality; ${ }^{4}$ the need to interrupt the compression for defibrillation; and rescuer fatigue, leading to inadequate manual chest compression. ${ }^{5}$ The guidelines recommend changing the rescuer every two minutes, ${ }^{3}$ which often results in interruption of the chest compressions.

Performing manual chest compressions in the catheter laboratory often leads to interruption in the procedure due to interference of the rescuer and the X-ray unit, and is associated with substantial technical difficulties and radiation exposure of the resuscitation staff.

In the past few years, different devices for automated chest compression or chest compression-decompression have been introduced. The LUCAS chest compression system (Physio-Control Inc/Jolife AB, Lund, Sweden) and the AutoPulse Load Distributing Band (ZOLL Medical Corporation, Chelmsford, MA, USA) are currently in use. The LUCAS device is a mechanical device that delivers sternal compression and decompression at a rate of around 100 per minute and a depth of around 4-5 $\mathrm{cm}$ by using a piston with a suction cup. Most of the parts in the LUCAS device are radiotranslucent. The first LUCAS-1 device was gas-driven, whereas the newer version, LUCAS-2, is electrically driven. 
An autopsy study has shown injuries after LUCAScardiopulmonary resuscitation (CPR) comparable to those seen after manual CPR. ${ }^{6}$ However, in non-autopsy studies, a few human and animal studies that have been conducted have shown that the device improved physiological data. ${ }^{7,8}$ In a recent randomized trial mechanical CPR compared with manual CPR did not result in improved 4-hour survival. ${ }^{9}$ Hence the use of mechanical resuscitation devices has not been introduced in the guidelines as routine treatment. ${ }^{3}$

In this case report, we present an ST-elevation myocardial infarction (STEMI) patient who survived prolonged inhospital cardiac arrest with good neurological outcome, with the use of an automatic compression-decompression device during percutaneous coronary intervention (PCI).

\section{Case summary}

A 50-year-old man, a smoker with hypertension, called the emergency service because of severe chest pressure, which woke him up in the night. He had a family history of coronary artery disease and was mildly obese. He had experienced two episodes of resting chest pain in the previous two days, each lasting around 20 minutes.

An electrocardiogram (ECG) taken in the ambulance revealed an inferior ST-elevation myocardial infarction with lateral ischemic changes. At ambulance arrival, the patient was awake and in severe pain, blood pressure was $60 \mathrm{mmHg}$ systolic, and pulse was $34 \mathrm{bpm}$. Oxygen saturation was not measured. Treatment with intravenous fluid was initiated. The ECG was transmitted to a hospital-based station inside the Coronary Care Unit and the patient was directly transported to the catheterization laboratory, which is the routine clinical practice in our hospital in cases of documented STEMI.

Directly after the patient's arrival to the catheterization laboratory, he suffered a cardiac arrest with the initial rhythm of ventricular fibrillation (VF). At first, he was successfully defibrillated to sinus, but shortly thereafter developed VF leading to an electrical storm. Chest compressions were initiated first manually but within minutes changed to the LUCAS-1 device. The LUCAS device is permanently located in our catheterization laboratory, and applying it to the patient takes less than a minute. The patient was intubated and ventilated. Good arterial pulses and oxygenation were achieved and these were monitored with an arterial line, which showed systolic pressure rising to $110 \mathrm{mmHg}$. After each defibrillation, the patient converted to nodal bradycardia but changed rhythm repeatedly to polymorph ventricular tachycardia (VT) and VF.

To treat bradycardia and possible bradycardia-triggered VT, an external transvenous pacemaker was inserted through the left femoral vein during PCI, resulting in good electrical capture during non-VT/VF episodes, but the insufficient blood pressure and the recurrent VF remained. Therefore, the automated CPR had to be continued. At this point, we decided to perform the coronary angiogram and PCI under ongoing CPR, hoping for stabilization of the so far therapy-resistant electrical storm. In order to secure good chest compressions and for technical feasibility of the PCI procedure, the LUCAS device was used during the whole procedure, which lasted for approximately 40 minutes (Supplementary video 1 ).

The coronary angiogram with the femoral approach revealed slow flow without apparent stenosis within the left coronary vessel and an occlusion of a large dominant mid right coronary artery. The culprit lesion was treated with a $4.0 \times 24 \mathrm{~mm}$ bare metal stent in the mid right coronary artery. The best working projection turned out to be an angulated view because of the LUCAS device, which has some radio-opaque components. The LUCAS device was paused shortly for stent deployment (Supplementary video 1 and Supplementary video 2).

There were no further ventricular arrhythmias seen after the PCI and the automatic compression device was turned off as the patient remained in sinus rhythm. Despite intravenous volume resuscitation and supposed adequate filling pressure, cardiac shock continued with a systolic blood pressure of 80-90 mmHg. Therefore, at this point an intra-aortic balloon pump (IABP) was placed and dopamine was started.

The patient was transferred to the intensive care unit for induced mild hypothermia treatment and ventilator therapy. Biochemical values revealed high cardiac markers and mild leukocytosis. Echocardiography on day 1 showed good left and right ventricular function with no wall movement abnormalities. After hypothermia treatment was induced to $32^{\circ} \mathrm{C}$, the patient was weaned from the ventilator and the IABP successfully. Adrenergic support with dopamine was also discontinued on day 2 . The patient was moved to the cardiac care unit for further medical treatment. He did not show neurological disability. There was no clinical suspicion of relevant rib or sternal fractures (we did not perform a computed tomography scan to look for asymptomatic fractures) and the patient did not experience any unusual extent of musculoskeletal chest pain. On day 10, he was discharged from hospital and returned home. His medications included clopidogrel, ASA (acetylsalicylic acid), beta-blocker, lipid-lowering drug, and angiotensin-converting-enzyme inhibitor.

At ambulatory clinical follow-up a few months later, the patient had stopped smoking and lost weight. He was asymptomatic and had no neurological problems (cerebral performance category scale 1). His blood pressure and cholesterol levels were within target range. He did an exercise 
test during the follow-up visit and had moderate exercise capacity but no ischemic changes or arrhythmias.

\section{Discussion}

This case is an example of a patient who had an in-hospital cardiac arrest due to acute myocardial infarction, complicated by cardiogenic shock. CPR using a mechanical chest compression device proved to give good perfusion pressure and made it possible to perform a PCI. The use of mechanical chest compression devices to facilitate PCI during cardiac arrest has been described in previous case reports. ${ }^{10,11}$

During manual chest compressions, a coronary angiography is very difficult to perform because the rescuer is a physical barrier for the rotational X-ray unit. The LUCAS device made it possible for the staff to concentrate on other treatment elements rather than solely on performing CPR. In the LUCAS device, the majority of the parts are radiolucent, which permits acceptable coronary imaging without risking high radiation exposure to the catheter laboratory staff. Apart from straight anterior-posterior projection, all standard angulations can be used. Angiography and wiring can be carried out with ongoing CPR and the device can easily be switched off for a few seconds for the exact stent placement.

The ventricular arrhythmias were not controllable with anti-arrhythmic drugs, external defibrillation, and a transvenous pacemaker. The electrical storm could only be causally managed by restoring blood flow in the right coronary artery. In our opinion, the compression device made the PCI possible. Treatment with extracorporeal membrane oxygenation was a considered possibility but was abandoned due to good hemodynamic response with help of the LUCAS and IABP. Although there is currently no evidence that the LUCAS device can reduce mortality compared to conventional CPR, we see an advantage in using the device in situations where effective, high-quality CPR is difficult to perform due to practical reasons (such as in a moving ambulance, during in-hospital transport, or in the catheterization laboratory). This, we believe, is particularly evident in cases like the one we present, where a potentially treatable cause to cardiac arrest has been identified.

\section{Conclusion}

Electrical storm in this case was managed with full neurological and cardiac recovery by giving efficient CPR using a mechanical CPR device and treating the underlying pathology, namely, acute coronary occlusion.

\section{Disclosure}

The authors have no conflicts of interest in this work.

\section{References}

1. Atwood C, Eisenberg MS, Herlitz J, Rea TD. Incidence of EMS-treated out-of-hospital cardiac arrest in Europe. Resuscitation. 2005;67(1): 75-80.

2. Paradis NA, Martin GB, Rivers EP, et al. Coronary perfusion pressure and the return of spontaneous circulation in human cardiopulmonary resuscitation. JAMA. 1990;263(8):1106-1113.

3. Koster RW, Baubin MA, Bossaert LL, et al. European Resuscitation Council Guidelines for Resuscitation 2010 Section 2. Adult basic life support and use of automated external defibrillators. Resuscitation. 2010;81(10):1277-1292.

4. Leary M, Abella BS. The challenge of CPR quality: improvement in the real world. Resuscitation. 2008;77(1):1-3.

5. Hightower D, Thomas SH, Stone CK, Dunn K, March JA. Decay in quality of closed-chest compressions over time. Ann Emerg Med. 1995;26(3):300-303.

6. Smekal D, Johansson J, Huzevka T, Rubertsson S. No difference in autopsy detected injuries in cardiac arrest patients treated with manual chest compressions compared with mechanical compressions with the LUCAS device - a pilot study. Resuscitation. 2009;80(10): 1104-1107.

7. Rubertsson S, Karlsten R. Increased cortical cerebral blood flow with LUCAS; a new device for mechanical chest compressions compared to standard external compressions during experimental cardiopulmonary resuscitation. Resuscitation. 2005;65(3):357-363.

8. Liao Q, Sjöberg T, Paskevicius A, Wohlfart B, Steen S. Manual versus mechanical cardiopulmonary resuscitation. An experimental study in pigs. BMC Cardiovasc Disord. 2010;10:53.

9. Rubertsson S, Lindgren E, Smekal D, et al. Mechanical chest compressions and simultaneous defibrillation vs conventional cardiopulmonary resuscitation in out-of-hospital cardiac arrest: the linc randomized trial. JAMA. 2014;311(1):53-61.

10. Ali A, Hothi SS, Cox D. Coronary intervention on a moving target: A case report and procedural considerations. $J$ Invasive Cardiol. 2013;25(8):E178-E179.

11. Wagner H, Terkelsen CJ, Friberg H, et al. Cardiac arrest in the catheterisation laboratory: A 5-year experience of using mechanical chest compressions to facilitate PCI during prolonged resuscitation efforts. Resuscitation. 2010;81(4):383-387.
Therapeutics and Clinical Risk Management

\section{Publish your work in this journal}

Therapeutics and Clinical Risk Management is an international, peerreviewed journal of clinical therapeutics and risk management, focusing on concise rapid reporting of clinical studies in all therapeutic areas, outcomes, safety, and programs for the effective, safe, and sustained use of medicines. This journal is indexed on PubMed Central, CAS,

\section{Dovepress}

EMBase, Scopus and the Elsevier Bibliographic databases. The manuscript management system is completely online and includes a very quick and fair peer-review system, which is all easy to use. Visit http://www.dovepress.com/testimonials.php to read real quotes from published authors. 